\section{Microcrédito formal y la sostenibilidad de las microempresas peruanas. Caso Lima}

\author{
Formal microcredit and the sustainability of Peruvian \\ microenterprises. Lima Case
}

\begin{abstract}
RESUMEN
Es de conocimiento público que Lima ha experimentado un fenómeno inmigratorio predominantemente desde la década de los 70's, evidenciándose en una explosión demográfica desordenada, donde la población de menores recursos ha encontrado en el emprendimiento popular, opción para el auto empleo y mejorar sus ingresos económicos y acceder a mejor calidad de vida, creando microempresas. El microcrédito formal apareció como forma de apoyo mutuo, entre la entidad aportante y la receptora. Esta investigación de enfoque cuantitativo, tipo aplicado, nivel relacional, diseño no experimental y corte transversal buscó determinar la relación entre microcrédito formal y la sostenibilidad de las microempresas peruanas, en el caso Lima Metropolitana, 2020. Se obtuvieron resultados descriptivos, de dispersión, de normalidad, y de prueba de hipótesis. Los resultados finales permitieron determinar que el microcrédito formal se relaciona positiva y moderadamente con la sostenibilidad (general, ambiental, social y económica) de las microempresas peruanas, específicamente en Lima, 2020. Por último, se presentan recomendaciones a representantes y tomadores de decisiones del gobierno, a directivos de empresas del sector bancario y a investigadores del área especializada en esta temática.
\end{abstract}

Palabras Clave: Banca; finanzas; microcrédito; sostenibilidad; formalidad.

\begin{abstract}
It is of public knowledge that Lima has experienced an immigration phenomenon predominantly since the 70's, evidenced by a disorderly demographic explosion, where the population of lesser resources has found in popular entrepreneurship, option for self-employment and improve their economic income and access to better quality of life, creating micro enterprises. The formal microcredit appeared as a form of mutual support, between the contributing and the receiving entity. This research, with a quantitative approach, applied type, relational level, non-experimental design and cross section, sought to determine the relationship between formal microcredit and the sustainability of Peruvian microenterprises, in the
\end{abstract}

\section{Pablo Willins Mauricio} Pachas

pmauriciop@unmsm.edu.pe ORCID: https://orcid.org/00000002-6453-0401

Universidad Nacional Mayor de San Marcos, Lima, Perú

Presentado: 27/10/2020 - Aceptado: 22/06/2021 - Publicado: 23/12/2021

(C) Los autores. Este artículo es publicado por la revista Gestión en el Tercer Milenio de la Facultad de Ciencias Administrativas Universidad Nacional Mayor de San Marcos. Este es un artículo de acceso abierto, distribuido bajo los términos de la licencia Creative Commons Atribución 4.0 Internacional (CC BY 4.0) [https://creativecommons.org/licenses/by/4.0/deed.es] que permite el uso, distribución y reproducción en cualquier medio, siempre que la obra original sea debidamente citada de su fuente original. 
case of Metropolitan Lima, 2020. Descriptive, dispersion, normality and hypothesis testing results were obtained. The results showed that formal microcredit is positively and moderately related to the sustainability (general, environmental, social and economic) of Peruvian microenterprises, specifically in Lima, 2020. Finally, recommendations are presented to government representatives and decision-makers, to managers of companies in the banking sector and to researchers specializing in this area.

Keywords: Banking; finance; microcredit; sustainability; formality.

\section{INTRODUCCIÓN}

En Lima, el fenómeno inmigratorio se dio en la década del 70 durante el gobierno militar de Velasco Alvarado, generándose una explosión demográfica en la capital y que de manera desordenada se crearon los asentamientos humanos donde se alojaron mayormente inmigrantes de menores recursos.

Esta situación conllevó a la búsqueda de ingresos para su subsistencia, surgiendo el autoempleo a través del comercio ambulatorio, talleres, actividades artesanales y diversos esfuerzos productivos. Fue en el año 1979 en que es redactada una nueva Constitución Política por parte de la Asamblea Constituyente, conteniendo un artículo en su Carta Magna que especifica claramente que el Estado es quien da el otorgamiento a las Municipalidades de autonomía y facultad de poner en marcha planes de creación de sus propias empresas para la generación de desarrollo en los niveles local y regional, a la vez que se promueve el ahorro comunal (Mauricio, 2004). En este marco constitucional, es que en el año 1980 se publica el Decreto Legislativo $\mathrm{N}^{\circ} 23039$, que genera marco legal para la creación de las cajas municipales, autorizándoles la captación de ahorros del público en provincias.

Surge así, la primera caja municipal en el país en el año 1982, llamada Caja Municipal de Ahorro y Crédito de Piura (CMAC Piura). Ésta tuvo como modelo las bancas comunales de países como España y Alemania, donde se encontraban desarrolladas, ofreciendo microcréditos formales como forma de apoyo mutuo, entre la entidad aportante y la receptora (Gómez et al., 2019).

De igual forma, en diversos países de Latinoamérica surgieron los microcréditos como alternativa de desarrollo regional (Cotler \& Rodríguez, 2008; Méndez et al., 2011; Romero et al., 2011; Sanhueza, 2013; Valentín, 2017).

El objetivo y la motivación de la microfinanciación es transformar las estructuras económicas rígidas y desarrollar la inclusión financiera proporcionando recursos financieros, como microcréditos y microahorros, a los hogares indigentes o pobres de las zonas rurales a fin de que puedan salir de la pobreza.

Como antecedente de investigación, podemos citar a Chirio y Álvarez (2017) que en su trabajo de tesis para obtener el título de licenciatura en Gestión por la Universidad Nacional San Agustín de Arequipa, plantearon como objetivo generar un análisis del impacto del microcrédito formal otorgado por la Caja Arequipa en el crecimiento de las Micro y Pequeñas Empresas en dicha ciudad. Concluyendo que en la Micro y Pequeñas Empresas (en adelante MYPES) existe una relación proporcional entre los montos de microcrédito recibidos y los niveles de ventas, utilidades y márgenes de utilidad sobre el costo. En segunda conclusión, se estableció que las MYPES del sector textil que lograron recibir microcréditos han logrado alcanzar mayores niveles de crecimiento, en comparación a aquellas que no han recibido microcrédito alguno.

Otro antecedente relevante, es el trabajo de Benites (2015), quien en su informe de tesis para obtener el título de Contador Público por la Universidad Señor de Sipán de Trujillo, planteó como objetivo determinar la influencia de las microfinanzas en el desarrollo empresarial de los comerciantes de un mercado. La investigación concluye aseverando que las Microfinanzas tienen efecto positivo y significativo en el desarrollo empresarial de los comerciantes en dicho contexto, validándose que mediante 
el otorgamiento de microcréditos, es posible el crecimiento económico integral de los negocios de los comerciantes, a la vez que se genera cambios favorables en sus vidas y las de sus familias. Sin embargo, es importante recalcar que el autor recomienda la promoción del Estado y las Instituciones Microfinancieras en la ejecución de programas de capacitación empresarial de MYPES frecuentemente, a fin de darles educación y sensibilización en cuanto a temática de gestión empresarial.

Como tercer antecedente, es necesario citar el trabajo de Linares (2016), quien en su trabajo de tesis para obtener el título de Economista por la Universidad Nacional de Trujillo, planteó como objetivo demostrar la influencia de las microfinanzas caracterizadas por el microcrédito en la rentabilidad de las MYPES del distrito de La Esperanza, en Trujillo. A través de análisis longitudinal, evaluando ratios de rentabilidad antes y después del uso de microcréditos, llegó a la conclusión que estos sí inciden en la mejora de los ratios de rentabilidad de las empresas evaluadas, logrando dar validez a su hipótesis.

Las tres investigaciones citadas líneas arriba son sólo algunas de las miles que existen a nivel mundial, sin embargo, no se encontró alguna que detalle específicamente la relación entre el microcrédito y la sostenibilidad de la empresa, a largo plazo, cuestión que motiva a investigadores como el caso nuestro, a realizar esta investigación.

En vista de los antecedentes planteados, en esta investigación se planteó el siguiente problema general de investigación:

¿El microcrédito formal se relaciona con la sostenibilidad de las microempresas peruanas, específicamente en Lima, 2020? Tratando de encarar la situación relevante del elevado números de entes informales que operan ofertando microcréditos a tasas de interés muy superiores a la que ofertan Instituciones financieras formales y que alcanzan tasas efectivas anuales de $213 \%, 790 \%, 1,300 \%$, deteriorando su escaso patrimonio y precarizando el trabajo. Ante un estado de postpandemia, hay que repensar en nuevas políticas económicas y financieras y responder a las revelaciones de la caótica situación del microempresario generador del autoempleo y la informalidad, ¿qué hacer de lo que no se ha hecho? y ¿qué no seguir haciendo?

Los siguientes problemas específicos fueron planteados:

- ¿El microcrédito formal se relaciona con la sostenibilidad ambiental de las microempresas peruanas, específicamente en Lima, 2020?

- ¿El microcrédito formal se relaciona con la sostenibilidad social de las microempresas peruanas, específicamente en Lima, 2020?

- ¿El microcrédito formal se relaciona con la sostenibilidad económica de las microempresas peruanas, específicamente en Lima, 2020?

De acuerdo con Hernández et al. (2014), todo proyecto de investigación debe ser justificado, a fin de demostrar las razones por la cual éste es emprendido. De acuerdo con Ñaupas et al. (2014), las justificaciones de la investigación pueden ser de carácter teórico, metodológico, práctico, entre otros.

Teóricamente, la investigación se justifica debido a que los resultados de la misma incrementarán el acervo bibliográfico de los temas planteados, y al ser publicados e indexados en las fuentes físicas y virtuales disponibles, ayudará a otros investigadores en la búsqueda de información actualizada de las variables tratadas en esta investigación.

Metodológicamente, la investigación se justifica debido a que el proceso de metodología, incluyendo las técnicas e instrumentos diseñados, podrá ser utilizado por otros investigadores a fin de realizar estudios similares, en otros contextos, como lo detalla Fernández (2020).

Prácticamente, la investigación se justifica por tratar de encarar la situación relevante de entes informales que otorgan préstamos a tasas efectivas anuales de $213 \%, 790 \%, 1,300 \%$, deteriorando el escaso patrimonio y precarizando el trabajo de las microempresas peruanas, en el problema detallado y sin solución en 
el año 2020, relacionado al microcrédito formal y la sostenibilidad de microempresas peruanas, en el contexto de Lima.

La investigación está alineada con los Objetivos de Desarrollo Sostenible al año 2030, propuesta por la Organización de las Naciones Unidas (ONU), específicamente este estudio tendrá impacto en el logro de los objetivos relacionados al fin de la pobreza, reducción de desigualdades, ciudades y comunidades sostenibles, producción y consumo responsables, y alianzas para el logro de los objetivos.

A nivel internacional, la investigación genera contribución al desarrollo de las líneas de investigación relacionadas a políticas de microcrédito formal y sostenibilidad empresarial, la cual tiene muy alta demanda hoy en día en el contexto académico.

A nivel nacional, los resultados de la investigación, tomando como punto de partida el caso de Lima, ayudará a sensibilizar y crear políticas efectivas, tomando como base el microcrédito formal, a fin de generar sostenibilidad (ambiental, social, económica) en las microempresas.

Para la Universidad Nacional Mayor de San Marcos, esta investigación logrará incrementar la producción científica, a la vez que le dará mayor reconocimiento y visualización, puesto que los resultados serán divulgados en revistas científicas, redes sociales y congresos internacionales.

De acuerdo con Bernal (2010), todo estudio cuantitativo de nivel correlacional debe contener hipótesis. Para Hernández et al. (2014), las hipótesis se clasifican en dos: general y específicas.

Hipótesis general de la investigación: el microcrédito formal se relaciona con la sostenibilidad de las microempresas peruanas, específicamente en Lima, 2020.

Hipótesis específicas: el microcrédito formal se relaciona con la sostenibilidad ambiental de las microempresas peruanas, específicamente en Lima, 2020; el microcrédito formal se relaciona con la sostenibilidad social de las microempresas peruanas, específicamente en Lima, 2020; y el microcrédito formal se relaciona con la sostenibilidad económica de las microempresas peruanas, específicamente en Lima, 2020.

En cuanto al objetivo general, este fue determinar la relación entre el microcrédito formal y la sostenibilidad de las microempresas peruanas, específicamente en Lima, 2020.

Los objetivos específicos fueron: determinar la relación entre el microcrédito formal y la sostenibilidad ambiental de las microempresas peruanas, específicamente en Lima, 2020; determinar la relación entre el microcrédito formal y la sostenibilidad social de las microempresas peruanas, específicamente en Lima, 2020; y determinar la relación entre el microcrédito formal y la sostenibilidad económica de las microempresas peruanas, específicamente en Lima, 2020.

\section{MÉTODOS}

La investigación contó con enfoque cuantitativo, de acuerdo con Hernández et al. (2014), este tipo de investigaciones se caracterizan porque pretenden medir la presencia de algún fenómeno. Los resultados del estudio son presentados en valores de frecuencias, porcentajes y coeficientes estadísticos.

Tipo aplicado, de acuerdo con Ñaupas et al. (2014), este tipo de investigación toma la información proveniente de investigaciones básicas (marco teórico) a fin de que sean utilizados como insumo para la construcción de instrumentos que permitan medir un fenómeno, para solucionar un problema delimitado.

El nivel fue relacional (o correlacional) y descriptivo. Es correlacional porque busca determinar la relación entre la variable 1 (microcrédito formal) y la variable 2 (sostenibilidad). Para Bernal (2010) este nivel de investigación es el más utilizado en las ciencias empresariales.

El diseño fue no experimental, para Hernández et al. (2014), este tipo de investigaciones se caracterizan por el hecho que el investigador a cargo no ejerce estímulo alguno sobre las variables, sino que las observa en su estado natural. Esta investigación analizará la relación entre el microcrédito formal y la sostenibilidad, sin ejercer estímulo alguno. 
El corte fue transversal, de acuerdo con Bernal (2010), este se caracteriza porque las observaciones son tomadas en una única vez, y no existen pruebas antes ni después (pre test, post test). La data será recolectada mediante el cuestionario una única vez.

Para el recojo de información, se utilizó un cuestionario elaborado por los autores y validado por cinco expertos (coeficiente de V. de Aiken $=0,98)$, además la confiabilidad del instrumento fue considerada buena (coeficiente de alfa de Cronbach $=0,897$ ).

La población es infinita, se desconoce el número exacto de encargados de microempresas peruanas (formales más informales), por lo que se utilizó la fórmula para el cálculo de muestra específico para estos casos.

La data recopilada en la prueba formal fue tratada en el software estadístico SPSS, donde se realizaron las siguientes pruebas:

- Resultados descriptivos.

- Resultados de dispersión

- Prueba de normalidad (Kolmogorov -Smirnov, al ser una muestra grande).

- Prueba de hipótesis (rho de Spearman, al ser variables cualitativas).

\section{RESULTADOS}

\section{Resultados descriptivos}

Los resultados descriptivos demuestran que, los promedios de las respuestas emitidas por los 384 sujetos que conformaron la muestra ascienden a 3,6839 para la variable microcrédito formal y 3,6885 para la variable sostenibilidad.
En cuanto a las dimensiones sostenibilidad ambiental, sostenibilidad social y sostenibilidad económica, se encontró que sus promedios ascienden a 3,6529, 3,7313 y 3,6781 respectivamente. Cabe resaltar que dichos resultados tuvieron como valor mínimo 1 , y como máximo 5 , por lo que su rango fue de 4 para cada variable y dimensión, tal y como se detalla en la Tabla 1.

\section{Gráfica de dispersión}

La Figura 1 muestra la gráfica de dispersión de las variables "microcrédito formal" y "sostenibilidad". Existe una clara línea de tendencia, la cual será analizada con mayor profundidad en los resultados de hipótesis.

\section{Prueba de normalidad}

Se procedió a realizar la prueba de normalidad estadística, con la finalidad de conocer la naturaleza de la data recolectada.

Todas las variables y dimensiones demostraron tener una significación asintótica bilateral menor a 0,05 , y por consiguiente permite al equipo de investigación determinar que los datos presentan distribución no normal, sugiriendo el uso de pruebas no paramétricas como rho de Spearman para las pruebas estadísticas de correlación, tal y como se muestra en la Tabla 2.

\section{Prueba de hipótesis.}

Hipótesis a contrastar:

Hipótesis general: El microcrédito formal se relaciona con la sostenibilidad de las microempresas peruanas, específicamente en Lima, 2020.

Hipótesis específica 1: El microcrédito formal se relaciona con la sostenibilidad ambiental

Tabla 1

Resultados descriptivos

\begin{tabular}{lccccccc}
\hline & N & Rango & Mínimo & Máximo & Media & Desv. típ. & Varianza \\
\hline Microcrédito Formal & 384 & 4,00 & 1,00 & 5,00 & 3,6839 &, 93323 &, 871 \\
Sostenibilidad & 384 & 4,00 & 1,00 & 5,00 & 3,6885 &, 78682 &, 619 \\
Sostenibilidad Ambiental & 384 & 4,00 & 1,00 & 5,00 & 3,6529 &, 89410 &, 799 \\
Sostenibilidad Social & 384 & 4,00 & 1,00 & 5,00 & 3,7313 &, 93756 &, 879 \\
Sostenibilidad Económica & 384 & 4,00 & 1,00 & 5,00 & 3,6781 &, 89754 &, 806 \\
\hline N válido (según lista) & 384 & & & & & &
\end{tabular}

Nota. Elaboración propia a partir de la aplicación del software SPSS versión 26 


\section{Figura 1}

Gráfica de dispersión

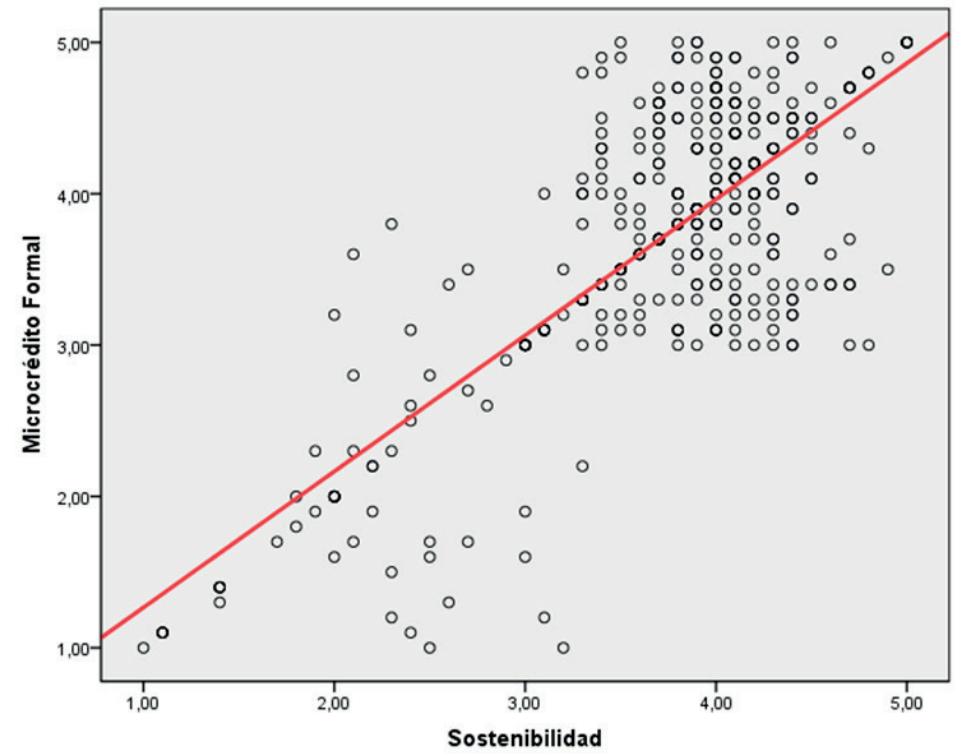

Tabla 2

Resultados de prueba de normalidad (Kolmogorov-Smirnov)

\begin{tabular}{llccccc}
\hline & & $\begin{array}{c}\text { Microcrédito } \\
\text { Formal }\end{array}$ & Sostenibilidad & $\begin{array}{c}\text { Sostenibilidad } \\
\text { Ambiental }\end{array}$ & $\begin{array}{c}\text { Sostenibilidad } \\
\text { Social }\end{array}$ & $\begin{array}{c}\text { Sostenibilidad } \\
\text { Económica }\end{array}$ \\
\hline $\mathrm{N}$ & 384 & 384 & 384 & 384 & 384 \\
Parámetros & Media & 3,6839 & 3,6885 & 3,6529 & 3,7312 & 3,6781 \\
normales & Desviación típica &, 93323 &, 78682 &, 89410 &, 93756 &, 89754 \\
& Absoluta &, 102 &, 162 &, 110 &, 095 &, 103 \\
Diferencias más & Positiva &, 079 &, 089 &, 066 &, 088 &, 070 \\
extremas & Negativa &,- 102 &,- 162 &,- 110 &,- 095 &,- 103 \\
\multirow{2}{*}{ Z de Kolmogorov-Smirnov } & 1,992 & 3,176 & 2,160 & 1,868 & 2,010 \\
\hline Sig. asintót. (bilateral) &, 001 &, 000 &, 000 &, 002 &, 001 \\
\hline
\end{tabular}

Nota. Elaboración propia a partir de la aplicación del software SPSS versión 26

de las microempresas peruanas, específicamente en Lima, 2020.

Hipótesis específica 2: El microcrédito formal se relaciona con la sostenibilidad social de las microempresas peruanas, específicamente en Lima, 2020.

Hipótesis específica 3: El microcrédito formal se relaciona con la sostenibilidad económica de las microempresas peruanas, específicamente en Lima, 2020.

A fin de dar validez o rechazo a las hipótesis planteadas, se hizo uso de la prueba estadística rho de Spearman, cuyos resultados se observan en la Tabla 3.
En cuanto a la hipótesis general: El microcrédito formal se relaciona con la sostenibilidad ambiental de las microempresas peruanas, específicamente en Lima, 2020; se encontró que existe correlación entre ambas variables, por cuanto la significación asintótica bilateral hallada fue de 0,000 (menor a 0,05 ), lo cual permite dar por válida la hipótesis general. Adicionalmente, es importante destacar que el coeficiente de correlación fue de 0,558, cuya interpretación permite a los investigadores determinar que la intensidad de la correlación es positiva moderada.

En cuanto a la hipótesis específica 1: El microcrédito formal se relaciona con la sos- 
Tabla 3

Resultados de pruebas de hipótesis (rho de Spearman)

\begin{tabular}{|c|c|c|c|c|c|c|c|}
\hline & & & $\begin{array}{c}\text { Microcrédito } \\
\text { Formal }\end{array}$ & Sostenibilidad & $\begin{array}{c}\text { Sostenibilidad } \\
\text { Ambiental }\end{array}$ & $\begin{array}{c}\text { Sostenibilidad } \\
\text { Social } \\
\end{array}$ & $\begin{array}{c}\text { Sostenibilidad } \\
\text { Económica }\end{array}$ \\
\hline \multirow{15}{*}{$\begin{array}{l}\text { Rho de } \\
\text { Spearman }\end{array}$} & \multirow{3}{*}{$\begin{array}{l}\text { Microcrédito } \\
\text { Formal }\end{array}$} & $\begin{array}{l}\text { Coeficiente de } \\
\text { correlación }\end{array}$ & 1,000 &, $558^{* *}$ &, $449^{* *}$ &, $535^{* *}$ &, $490^{* *}$ \\
\hline & & Sig. (bilateral) & . & ,000 & ,000 & ,000 &, 000 \\
\hline & & $\mathrm{N}$ & 384 & 384 & 384 & 384 & 384 \\
\hline & \multirow{3}{*}{ Sostenibilidad } & $\begin{array}{l}\text { Coeficiente de } \\
\text { correlación }\end{array}$ &, $558^{* *}$ & 1,000 &, $789^{* *}$ &, $789^{* *}$ &, $758^{* *}$ \\
\hline & & Sig. (bilateral) & ,000 & . & ,000 & ,000 & ,000 \\
\hline & & $\mathrm{N}$ & 384 & 384 & 384 & 384 & 384 \\
\hline & \multirow{3}{*}{$\begin{array}{l}\text { Sostenibilidad } \\
\text { Ambiental }\end{array}$} & $\begin{array}{l}\text { Coeficiente de } \\
\text { correlación }\end{array}$ &, $449^{* *}$ &, $789^{* *}$ & 1,000 &, $481^{* *}$ & , 458 \\
\hline & & Sig. (bilateral) & ,000 & ,000 & . & ,000 & ,000 \\
\hline & & $\mathrm{N}$ & 384 & 384 & 384 & 384 & 384 \\
\hline & \multirow{3}{*}{$\begin{array}{l}\text { Sostenibilidad } \\
\text { Social }\end{array}$} & $\begin{array}{l}\text { Coeficiente de } \\
\text { correlación }\end{array}$ &, $535^{* *}$ &, $789^{* *}$ &, $481^{* *}$ & 1,000 &, $433^{* *}$ \\
\hline & & Sig. (bilateral) &, 000 & ,000 &, 000 & $\cdot$ &, 000 \\
\hline & & $\mathrm{N}$ & 384 & 384 & 384 & 384 & 384 \\
\hline & \multirow{3}{*}{$\begin{array}{l}\text { Sostenibilidad } \\
\text { Económica }\end{array}$} & $\begin{array}{l}\text { Coeficiente de } \\
\text { correlación }\end{array}$ & $490^{* *}$ &, $758^{* *}$ &, $458^{* *}$ &, $433^{* *}$ & 1,000 \\
\hline & & Sig. (bilateral) & ,000 & ,000 & ,000 & ,000 & . \\
\hline & & $\mathrm{N}$ & 384 & 384 & 384 & 384 & 384 \\
\hline
\end{tabular}

Nota. Elaboración propia a partir de la aplicación del software SPSS versión 26

tenibilidad ambiental de las microempresas peruanas, específicamente en Lima, 2020; se encontró que existe correlación entre ambas variables, por cuanto la significación asintótica bilateral hallada fue de 0,000 (menor a 0,05), lo cual permite dar por válida la hipótesis general. Adicionalmente, es importante destacar que el coeficiente de correlación fue de 0,449 , cuya interpretación permite a los investigadores determinar que la intensidad de la correlación es positiva moderada.

En cuanto a la hipótesis específica 2: El microcrédito formal se relaciona con la sostenibilidad social de las microempresas peruanas, específicamente en Lima, 2020; se encontró que existe correlación entre ambas variables, por cuanto la significación asintótica bilateral hallada fue de 0,000 (menor a 0,05), lo cual permite dar por válida la hipótesis general. Adicionalmente, es importante destacar que el coeficiente de correlación fue de 0,535 , cuya interpretación permite a los investigadores determinar que la intensidad de la correlación es positiva moderada.

En cuanto a la hipótesis específica 3: El microcrédito formal se relaciona con la sostenibilidad económica de las microempresas peruanas, específicamente en Lima, 2020; se encontró que existe correlación entre ambas variables, por cuanto la significación asintótica bilateral hallada fue de 0,000 (menor a 0,05), lo cual permite dar por válida la hipótesis general. Adicionalmente, es importante destacar que el coeficiente de correlación fue de 0,490, cuya interpretación permite a los investigadores determinar que la intensidad de la correlación es positiva moderada.

\section{DISCUSIÓN}

En el trabajo presentado por Chirio y Álvarez (2017), se estableció que las MYPES textiles que han lograron recibir microcréditos formales alcanzaron mayores niveles de crecimiento frente a aquellas que no; en este sentido, los resultados de esta investigación respaldan de manera científica la propuesta de los autores, por cuanto existe relación entre el microcrédito formal y la sostenibilidad de las microempresas peruanas.

Asimismo, el trabajo de investigación realizado por Benites (2015) logró determinar la influencia de las microfinanzas en la mejora y desarrollo empresarial de comerciantes en un centro de abasto, encontrándose que mediante 
los microcréditos adquiridos, diversas MYPES logran crecer de manera económica e integral, logrando de esta manera ciertos cambios favorables en sus vidas y las de sus familias. Estos resultados respaldan lo propuesto por Benites (2015), es importante recalcar que el autor recomienda la promoción del Estado y las Instituciones Microfinancieras en la ejecución de programas de capacitación empresarial de MYPES frecuentemente, a fin de darles educación y sensibilización en cuanto a temática de gestión empresarial.

Los resultados presentados por Linares (2016) lograron demostrar la influencia de las microfinanzas caracterizadas por el microcrédito en la rentabilidad de las MYPES del distrito de La Esperanza, en Trujillo. Este trabajo de investigación logró obtener similares resultados, pero en otro contexto (Lima), permitiendo complementar la teoría relacionada al tema y obtener un diagnóstico más amplio de esta problemática empresarial.

\section{CONCLUSIONES}

- El microcrédito formal se relaciona positiva y moderadamente con la sostenibilidad de las microempresas peruanas, específicamente en Lima, 2020 (p. $=0,000 ; r=0,558)$.

- El microcrédito formal se relaciona positiva y moderadamente con la sostenibilidad ambiental de las microempresas peruanas, específicamente en Lima, 2020 (p. =0,000; r = 0,449).

- El microcrédito formal se relaciona positiva y moderadamente con la sostenibilidad social de las microempresas peruanas, específicamente en Lima, 2020 (p. =0,000; r = 0,535).

- El microcrédito formal se relaciona positiva y moderadamente con la sostenibilidad económica de las microempresas peruanas, específicamente en Lima, 2020 (p. =0,000; r = 0,490).

Se recomienda a representantes y tomadores de decisiones del gobierno a crear políticas que permitan promover e incentivar el microcrédito entre los empresarios peruanos, puesto que el acceso al mismo está relacionado moderadamente con la sostenibilidad que puedan alcanzar sus empresas.

Se recomienda a investigadores realizar investigaciones similares que permitan continuar con la línea de investigación propuesta, a fin de incrementar la teoría relacionada al tema.

Se propone la promoción del microcrédito formal a través del impulso de cajas comunitarias o populares, en comunidades provinciales y distritales del País. Su creación, podrá ser fomentada por las cámaras de comercio o asociaciones de microempresarios, emprendedores individuales, totalmente autónomas, con asistencia técnica de parte del estado a través de la superintendencia de banca y seguros, para emprender sus operaciones crediticias y coberturas de prevenciones sociales, mediante sistema de juntas y con periodo de gracia tributaria, exonerándoles los primeros cinco años. Este sistema y mecanismos financieros tendrán un impacto directo en la sostenibilidad de las microempresa y en la reducción de la informalidad).

Se propone que el Estado impulse programas de adquisiciones de bienes y servicios de microempresas formales, con asistencia técnica de parte del estado a través de la superintendencia de Registros Públicos promueva la formalización y un área estatal de promoción empresarial.

\section{REFERENCIAS BIBLIOGRÁFICAS}

Benites, M. (2015). Influencia de las microfinanzas en el desarrollo empresarial de los comerciantes del mercado José Quiñones Gonzales de la ciudad de Chiclayo - 2015 (Tesis de Grado). Universidad Señor de Sipán. Perú. http://repositorio.uss. edu.pe/handle/uss/3025

Bernal, C. (2010). Metodología de la investigación. (3.a ed.). Bogotá, Colombia: Pearson Educación.

Chirio, K., y Álvaez, R. (2017). Impacto del microcrédito en el crecimiento de las micro y pequeñas empresas textiles atendidas por la Caja Arequipa, 2016 (Tesis de Grado). Universidad Nacional de San Agustín de Arequipa. Perú. http://repositorio.unsa.edu.pe/handle/UNSA/4340

Cotler, P., y Rodríguez, E. (2008). Rentabilidad y tamaño de préstamo de las microfinanzas en México: Un estudio de caso. Economía mexicana. 
Nueva época, 17(2), 149-169. http://www.scielo.org.mx/pdf/emne/v17n2/v17n2a1.pdf

Fernández, V. H. (2020). Tipos de justificación en la investigación científica. Espíritu Emprendedor TES, 4(3), 65-76. https://doi.org/10.33970/eetes.v4.n3.2020.207

Gómez, G., Mena, A., y Beltrán, R. (2019). Estudio de los factores determinantes del crecimiento de las cajas municipales de ahorro y crédito del Perú. Contaduría y administración, 64(3), 1-19. https://doi.org/10.22201/ fca.24488410e.2018.1596

Hernández, R., Fernández, C., y Baptista, P. (2014). Metodología de la investigación. (6.a ed.). México D.F., México: McGraw-Hill.

Linares, H. (2016). Incidencia del microcrédito en la rentabilidad de las MYPES del distrito de La Esperanza, periodo 2010:2014 (Tesis de Grado). Universidad Nacional de Trujillo. Perú. http:// dspace.unitru.edu.pe/handle/UNITRU/5157

Mauricio, P. (2004). Préstamos caros a las PYMES; le restan competitividad. Gestión en el Tercer Milenio, 7(13), 19-27. https://doi.org/10.15381/ gtm.v7i13.9803

Méndez, J., Hernández, H., y Stella, N. (2011). Evaluación del impacto de las Microfinanzas sobre los ingresos y la generación de empleo en Colombia. Cuadernos de Administración, 27(46), 81-89. http://www.scielo.org.co/pdf/cuadm/v27n46/ v27n46a07.pdf
Ñaupas, H., Mejía, E., Novoa, E. y Villagómez, A. (2014). Metodología de la investigación. (4.a ed.). Bogotá, Colombia: Ediciones de la U.

Romero, Y., Arenas, A., Carrillo, D., y Vergara, D. (2011). Aspectos del Microcrédito en su Concepción Original, en Búsqueda de Factores de Mayor Impacto en Colombia: Revisión de Tema. TecnoLógicas, 26(1), 113-132. http://www.scielo.org.co/pdf/teclo/n26/n26a07.pdf

Sanhueza, P. (2013). Las Microfinanzas como Instrumento de Apoyo al Sector Microempresarial en Chile: Estado y Desafíos. Journal of technology management \& innovation, 8(2), 209-220. http://dx.doi.org/10.4067/S071827242013000200017

Valentín, L. (2017). Desarrollo local y microfinanzas como estrategias de atención a las necesidades sociales: un acercamiento teórico conceptual. Revista mexicana de ciencias políticas y sociales, 62(299), 101-128. http://www.scielo.org.mx/pdf/rmcps/v62n229/0185-1918rmcps-62-229-00101.pdf 